Sehi Antoine Mian Bi Université virtuelle de Côte d'Ivoire

(Côte d'Ivoire)

\section{Usages du groupe Facebook en situation de stage : le cas des éducateurs de l'ENS d'Abidjan}

doi: $10.18162 / f p .2018 .488$

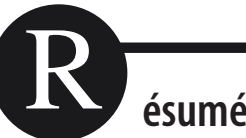

Cette recherche a pour objectif de mieux comprendre les usages d'un groupe du réseau social Facebook par des étudiants dans le cadre de leur stage pratique. La collecte et l'analyse des données se sont basées sur une méthodologie qualitative par entretiens semi-directifs et sur une méthodologie quantitative par questionnaire. Les résultats montrent que le groupe Facebook est un nouvel espace de socialisation qui permet aux stagiaires de briser l'isolement en favorisant les relations interpersonnelles. En effet, le groupe leur permet d'avoir des informations sur la formation, de garder le contact avec leurs collègues, d'avoir du soutien et de partager des expériences de terrain.

\section{Mots-clés}

Réseaux sociaux, École normale supérieure d'Abidjan, usage de Facebook, stage, Côte d'Ivoire.

\section{Abstract}

This research aims to better understand the uses of a Facebook social network group by students as part of their internship. Data collection and analysis were based on qualitative and quantitative methodology through semistructured interviews and survey. The results show that the Facebook group is a new social space that allows trainees to break the isolation by consolidating interpersonal relationships. Indeed, the group allows them to have information on training, to keep in touch with their colleagues, to have support and to share experiences in the field.

\section{Keywords}

Social networks, ENS Abidjan, Facebook use, Placement, Côte d'Ivoire.

\section{Introduction}

Longtemps considérés comme outils ludiques pour entretenir des relations amicales et passer le temps (Thivierge, 2011), les réseaux sociaux, et précisément Facebook, nont cessé d'évoluer, ouvrant d'autres possibilités d'usage en contexte de formation (Peraya et Bonfils, 2014). En effet, comme le reconnaissent Loiseau, Potolia et Zourou (2011), ce site de réseautage social ouvrira de nouvelles pistes dans l'apprentissage des langues, par exemple, en raison de son potentiel collaboratif. En présentant Facebook comme une vaste plateforme d'interactions, Diakhaté et Akam (2015) montrent qu'il facilite la communication entre ses abonnés et permet le partage de différents types de contenus (vidéos, photos, fichiers divers, etc.). En mettant à profit le potentiel collaboratif de Facebook, des recherches ont montré comment des étudiants ont intégré cet outil dans leurs pratiques d'apprentissage en formation initiale dans le contexte aussi bien africain (Ben Rebah et Dabove, 2017; Diakhaté et Akam, 2015; Mian Bi, 2012) qu'européen (Peraya et Bonfils, 2014).

La présente étude, qui a pour cadre la formation initiale des éducateurs en Côte d'Ivoire, vise à mieux comprendre les usages d'un groupe Facebook par des étudiants de l'ENS d'Abidjan dans le cadre de leur stage pratique.

\section{Contexte et justification}

À l'instar de la majorité des pays d'Afrique, les usages sans cesse évolutifs des TIC influencent non seulement les pratiques communicationnelles, mais aussi les pratiques économiques et sociales des habitants de la Côte d'Ivoire (Loukou, 2015). Et comme un peu partout en Afrique (Tchameni Ngamo, 2007), les TIC sont de plus en plus présentes dans la société ivoirienne (Bogui, 2007). Les chiffres 
de l'Autorité de régulation des télécommunications de Côte d'Ivoire (ARTCI) montrent que le taux de pénétration du téléphone mobile est de plus de 100 \%. En 2017, la Côte d'Ivoire comptait près de 10490000 millions d'utilisateurs d'internet sur une population totale de 22671331 d'habitants. La grande majorité des utilisateurs (10 401 187) sont des abonnés à internet mobile. Selon le ministère de l'Économie numérique et de la Poste, la Côte d'Ivoire compte aussi 3 millions d'utilisateurs de Facebook.

À travers la mise en ouvre du projet e-Éducation et de la réforme Licence Master Doctorat (Krou Adohi, 2012), le système éducatif ivoirien a franchi un palier dans sa volonté d'intégrer les TIC. Même si les institutions d'enseignement de façon générale, et particulièrement celles du supérieur, ne sont pas équipées en matériel (Loukou, 2015; Mian Bi, 2016), les TIC sont intégrées comme une matière transversale dans les maquettes pédagogiques (Mian Bi, 2016). À l'ENS d'Abidjan, depuis la rentrée universitaire 2012, la formation initiale du personnel d'appui technique à l'éducation (PATE), anciennement appelé éducateur, s'est vue renforcée avec un volume horaire de 50 heures pour les TICE, contre 10 heures dans l'ancien programme de formation (Mian Bi, 2016). Comme tous les concours d'entrée à l'ENS d'Abidjan (Koffi,2012), l'accès à la formation de PATE se fait par concours direct pour ceux n'ayant pas de statut de fonctionnaire et par concours professionnel pour les fonctionnaires. Ainsi, la population estudiantine en formation initiale des PATE comprend aussi bien des fonctionnaires en reprise de formation que des étudiants ayant un parcours plus rectiligne de formation. Il faut mentionner que cette formation initiale des éducateurs se déroule sur deux années, dont la première, in situ, est théorique et la seconde, pratique, sous forme de stage dans les établissements d'enseignement secondaire.

Les fonctionnaires en reprise de formation et inscrits en formation initiale à l'ENS éprouvent des difficultés comme il est mentionné par Koffi (2012). Dans son étude portant sur 13 stagiaires en formation initiale à l'ENS, l'auteur a identifié six difficultés rencontrées par ces derniers dans le cadre de leur formation. La première porte sur le concours professionnel d'entrée à l'ENS d'Abidjan auquel sont soumis ces fonctionnaires en poste. Pour favoriser leur réussite à ce concours organisé par le ministère de la Fonction publique, certains d'entre eux ont suivi des cours de préparation en plus d'être inscrits pour suivre des cours de formation continue. La seconde est liée à l'ENS et s'intéresse essentiellement sur leur adaptation à leur nouvelle vie d'étudiant. Les troisième et quatrième difficultés, qui sont d'ordre pédagogique, concernent l'apprentissage et la gestion du temps. Les deux dernières difficultés sont d'ordre socioéconomique et portent sur la gestion de la famille et des questions financières du fait de leur nouvelle vie. Dans le cadre de la présente étude, nous nous sommes intéressé aux difficultés d'apprentissage qui peuvent être rencontrées par ces fonctionnaires en reprise de formation, mais aussi par les étudiants non fonctionnaires. En effet, les enseignements à l'ENS d'Abidjan étant plus transmissifs et centrés sur l'enseignant comme l'a mentionné l'étude de Koffi (2007), ces mêmes difficultés d'apprentissage peuvent être rencontrées par les étudiants non fonctionnaires. Selon l'auteur, les difficultés d'apprentissage rencontrées par ces fonctionnaires en reprise de formation portent sur la mémorisation des notes de cours qui requiert assez de concentration. Il y a aussi la question de la prise de notes, puisque la plupart des enseignants ne mettent pas à la disposition des étudiants des supports de cours. En plus des difficultés liées à l'apprentissage, en formation in situ, relevées par Koffi (2012), on peut ajouter les conditions souvent difficiles du déroulement des stages pratiques. En effet, à la fin de leur première année de formation in situ à Abidjan, les étudiants sont envoyés en stage dans 
des établissements d'enseignement secondaire dans les villes de l'intérieur du pays. En l'absence de dispositifs institutionnels pour favoriser les interactions entre étudiants en stage, ces derniers éprouvent d'énormes difficultés pour réduire leur isolement, se soutenir mutuellement, échanger des expériences, des informations et discuter de leur formation. En effet, comme l'a mentionné Asselin (2002) dans sa recherche, du fait de l'absence de lien constant et sécurisant avec leurs compagnons de classe, les étudiants en stage peuvent éprouver des sentiments d'isolement et d'abandon.

Pour pallier le manque d'infrastructures TIC dans les universités, les étudiants font de plus en plus usage de leurs équipements personnels. En 2015, une étude de Mian Bi (2016) sur une population de 166 éducateurs en première année de formation à l'ENS d'Abidjan a montré que la totalité disposait d'un téléphone mobile et 43,7\% d'un ordinateur. Si 57,8\% affirmaient accéder à internet dans un cybercafé, $51,8 \%$ y accédaient via les terminaux mobiles. Fortement présents $(87,9 \%)$ sur les réseaux sociaux, ces éducateurs en formation initiale étaient dans leur grande majorité inscrits sur Facebook (87,6\%). Comme l'ont montré les travaux de Attenoukon, Coulibaly et Karsenti (2016) au Bénin, le manque d'infrastructures TIC pour la formation à l'ENS d'Abidjan fait que les seuls moyens de communication entre les étudiants sont, en plus du téléphone mobile, les médias sociaux tels que Facebook Messenger et WhatsApp. Par ailleurs, les travaux de Mian Bi $(2012,2016)$ avaient montré aussi que, dans le cadre de leur formation initiale, les éducateurs avaient utilisé de leur propre initiative un groupe Facebook pour partager des contenus de formation.

Dans la littérature scientifique, on rencontre plusieurs façons d'utiliser Facebook dans l'enseignement supérieur. Les étudiants l'utilisent dans le cadre de leurs devoirs (Mian Bi, 2012), dans le cadre de leurs apprentissages pour la co-construction dynamique de connaissances (Diakhaté et Akam, 2015). Il peut aussi être utilisé comme outil en formation à distance (Ben Rebah et Dabove, 2017; Mélot, Strebelle, Mahauden et Depover, 2016; Peraya et Bonfils, 2014). Par ailleurs, Mélot et al. (2016) montraient dans leur étude que, grâce à Facebook, les étudiants sont capables de développer leur habileté à communiquer. Ben Rebah et Dabove (2017) vont dans le même sens et affirment que, comme outil de partage, de communication et d'échanges, Facebook favorise aujourd'hui l'interaction entre les apprenants.

Alors que les études sur les usages des médias sociaux en formation sont peu nombreuses en Côte d'Ivoire, la présente étude, qui s'inscrit dans le prolongement de l'étude de Mian Bi (2016), vise à mieux comprendre les usages que font ces étudiants du groupe Facebook pendant leur stage.

\section{Cadre théorique}

L'approche instrumentale développée par Rabardel (1995) nous semble assez pertinente pour servir d'appui théorique à notre étude. En effet, selon cet auteur, l'homme est entouré d'artefacts et de technologies culturellement constitués qu'il peut mobiliser au cours de son activité afin d'atteindre son objectif. Mais ceux-ci ne deviennent instruments que lorsqu'ils jouent le rôle de médiateur entre le sujet et l'objet. Pour Rabardel (1995), un objet technique n'est pas d'emblée un instrument, mais une proposition qui sera développée ou non par un utilisateur. C'est lorsque cet instrument est transformé dans l'activité par son utilisateur en fonction d'un usage construit par celui-ci qu'il deviendra instrument. Ainsi, l'artefact associé au geste qui le rend efficace constitue l'instrument. Et c'est à travers l'usage que se constitue progressivement une organisation invariante de l'action, un schème. 
Dans cette approche, l'instrument, défini comme une entité mixte, est constitué d'un artefact, la forme matérielle de l'objet matériel (Plantard, 2016) et de schèmes considérés comme la composante psychologique du geste (Nogry, Decortis, Sort et Heurtier, 2013; Plantard, 2016; Vergnaud, 1991).

Selon Rabardel (1995), l'instrument peut avoir différentes fonctions et constituer un médiateur dans différents types de relations et de directions : vers l'objet de l'activité, vers soi et vers les autres. Comme médiateur de la relation entre sujet et objet de l'activité,l'instrument peut permettre de transformer cet objet (médiation pragmatique) ou de construire des connaissances sur celui-ci (médiation épistémique). L'instrument peut par ailleurs permettre de réguler l'activité propre de la personne (médiation réflexive), ou de supporter la communication et la collaboration entre les acteurs de l'activité (médiation interpersonnelle).

Dans la présente étude, nous nous sommes intéressé à la médiation interpersonnelle, à travers un réseau social numérique comme instrument. En effet, le monde universitaire semble s'approprier des environnements sociotechniques, comme les réseaux sociaux numériques qui offrent aujourd'hui l'opportunité de tisser de nouvelles formes de sociabilité et de partage. Pour Petiau (2011), ces nouveaux médias sont d'abord de nouvelles modalités techniques pour entretenir les liens, qui prolongent la sociabilité telle qu'elle peut se tenir dans d'autres espaces. Dans ses travaux, Akoun (2002) mentionnait déjà qu'avec ces instruments de l'internet, les modes de communication sont transformés puisqu'on est à distance de chacun et proche de tous. Petiau (2011) et Loisier (2014) mettent en exergue que les réseaux sociaux numériques offrent un grand potentiel de multiplication des relations sociales, ce qui, en réduisant virtuellement la distance entre les individus, permet à chacun de s'adresser à un groupe plus large. Toutefois, même s'il en montre l'importance, Loisier (2014) met en garde contre la théâtralisation des adeptes des réseaux sociaux qui consiste à mettre en vedette sa vie quotidienne censée être remarquable.

Utilisés souvent soit pour entretenir les relations amicales et faire passer le temps (Thivierge, 2011), soit comme instruments pour la co-construction dynamique de connaissances chez les étudiants (Diakhaté et Akam, 2015; Mian Bi, 2012), soit pour l'apprentissage collaboratif à l'université (Chomienne et Lehmans, 2012), les réseaux sociaux semblent de plus en plus incontournables pour l'intégration sociale (Tinto, 1975) dans le monde universitaire aussi bien en formation initiale qu'en contexte de stage pratique. Dans son modèle initial, Tinto (1975) définit l'intégration sociale comme l'interaction entre l'étudiant et les différents intervenants du milieu universitaire. Dans leur recherche qui s'est attachée à valider le modèle initial de Tinto (1975), Berger et Milem (1999) ont montré que la perception de l'environnement de soutien inclut le soutien des pairs qui renvoie au fait de se sentir à l'aise ou en confiance avec d'autres étudiants, de partager des opinions et des valeurs avec d'autres étudiants et d'avoir l'opportunité de développer des amitiés. Si, dans le contexte des universités du Nord, l'utilisation des réseaux sociaux est souvent une initiative institutionnelle, en Afrique, elle est généralement le fait d'un groupe d'étudiants (Attenoukon et al., 2016; Mian Bi, 2012). Des travaux de Mian Bi (2012), il ressort que ces étudiants initiateurs sont généralement motivés par le fait de trouver un espace de communication. Après avoir créé le groupe, les initiateurs sensibilisent leurs collègues afin qu'ils l'intègrent. Pour la bonne marche du groupe, des règles d'utilisation sont mises en place de façon informelle et des modérateurs sont désignés. 
Les travaux sur les usages des TIC durant les stages de fin d'études abondent aujourd'hui dans la littérature scientifique. Laferrière et al. (2001) montraient déjà dans leurs études que l'usage des TIC par les stagiaires favorisait la collaboration entre eux. Dans le même ordre d'idées, Nault et Nault (2001) ont montré dans leur recherche que l'utilisation de la plateforme WebTC par des étudiants durant le stage leur permettait de s'épauler. Citant d'autres expériences, ces chercheures estiment qu'avoir la chance d'échanger des commentaires sur leurs expériences, grâce aux TIC, pourrait être un moyen de briser l'isolement chez les stagiaires. En complément des supervisions directes, les travaux d'Asselin (2002) se sont aussi penchés sur l'utilisation des outils virtuels de communication de l'environnement pédagogique WebCT durant le stage. L'un des principaux avantages mentionnés par les étudiants dans cette étude est le fait de pouvoir communiquer leurs idées et de pouvoir garder le contact avec le groupe. Comme Asselin (2002), Karsenti, Lepage et Gervais (2002) ont mené une étude pour mieux comprendre les interactions favorisées par le forum de discussion et le groupe électronique de discussion sur la supervision et l'accompagnement des stages. Les résultats de cette étude ont montré que ces outils virtuels de communication représentent un mode de soutien essentiel. Tout comme les travaux d'Asselin (2002) et de Karsenti et al. (2002), ceux de Mayer (2002) mettent en évidence le potentiel des TIC qui fournit aux stagiaires de nouveaux espaces d'apprentissage permettant un réseautage ainsi qu'un soutien personnel et professionnel. En se basant sur la théorie de l'activité, Gérard (2007) a mis en exergue dans ses travaux qu'en contexte de stage, le réseau social virtuel permet à certains stagiaires d'être considérablement plus intégrés et d'avoir un réseau social beaucoup plus important que le réseau social en présentiel. En plus d'une intégration accrue du stagiaire, le réseau social virtuel développe son employabilité comme le montrent les résultats d'une enquête de $\mathrm{He}$, Gu, Wu, Zhai et Song (2017) menée auprès de 196 étudiants diplômés en Chine. Selon ces auteurs, l'utilisation des médias sociaux est positivement liée aux compétences relatives à l'employabilité. Pour eux, l'efficacité des stages sert de mécanisme de médiation par lequel l'utilisation des médias sociaux affecte les compétences d'employabilité.

Le contexte de la formation des éducateurs à l'ENS d'Abidjan est marqué par l'absence de dispositifs qui favorisent l'interaction des étudiants une fois en stage. Pour faire face à cet état de fait, il semble aujourd'hui pertinent de mettre en œuvre des environnements familiers aux étudiants et qui favorisent l'interaction afin de renforcer des moyens déjà utilisés par les étudiants en stage avec des visées formatrices. En première année de formation, in situ à l'ENS d'Abidjan, des étudiants avaient créé un groupe Facebook (Mian Bi, 2016). Nous voulons, à travers la présente étude, comprendre les usages que font ces étudiants de ce groupe, comme instrument pour la médiation interpersonnelle (Rabardel, 1995), dans le cadre de leurs stages pratiques qui ont lieu dans des établissements d'enseignement secondaire.

\section{Méthodologie}

La population cible est celle des éducateurs de la promotion 2014-2016 en stage inscrits dans un groupe Facebook. Dès que nous avons eu connaissance de son existence, étant leur enseignant en initiation à l'informatique, nous avons fait une demande aux administrateurs dudit groupe pour y être inscrit. Ainsi, depuis la première année de formation, nous avons pu suivre les échanges entre les étudiants et nous sommes souvent intervenu dans les débats lorsque les étudiants demandaient notre 
avis ou mentionnaient notre nom dans des publications. Lorsque nous avons commencé les recherches dans le cadre de cette étude, ce groupe comptait près de 200 étudiants, tous de la promotion 20142016. Pour mieux comprendre les usages que font ces étudiants du groupe Facebook lors de leurs stages, nous avons adopté une démarche méthodologique quantitative (questionnaire) et qualitative (guide d'entretien semi-dirigé). Ainsi, pour la présente étude, deux instruments ont été utilisés pour la collecte des données. Le questionnaire, qui a été adressé à tous les éducateurs, comportait neuf questions: quatre questions à choix multiples et cinq questions ouvertes. Les questions à choix multiples ont porté sur le lieu de stage, les équipements utilisés pour accéder au groupe Facebook et les sujets abordés dans le groupe. Pour identifier ces sujets, nous nous sommes basé sur les catégories des messages envoyés dans le groupe de discussion issues des travaux de Karsenti et al. (2002) et des travaux de Mian Bi (2012) : soutien et encouragement mutuel, partage d'expérience, accès à l'information, renforcement des acquis académiques, rencontre et échanges, maintien du contact et tous les autres types de messages qui n'intègrent pas les catégories citées plus haut. Les questions ouvertes devraient nous permettre d'illustrer les sujets abordés. Le guide d'entretien semi-dirigé a été adressé aux deux initiateurs du groupe Facebook. Les points abordés dans ce guide sont issus des travaux de Mian Bi (2012) et portaient sur la motivation pour la création du groupe, l'existence d'un code de conduite, la modération du groupe et l'importance du groupe pendant le stage.

Pour la passation du questionnaire, nous avons, à travers les initiateurs du groupe, lancé un avis dans le groupe Facebook auquel seulement 32 étudiants en stage ont répondu favorablement. En ce qui concerne le guide d'entretien, nous avons organisé une rencontre avec les deux initiateurs du groupe Facebook. L'entretien avec les deux membres qui ont créé le groupe a été enregistré et retranscrit.

Pour le traitement des données quantitatives collectées à partir des questions à choix multiples, l'analyse statistique est plutôt descriptive. En ce qui concerne les données qualitatives récoltées à travers les questions ouvertes et le guide d'entretien semi-dirigé, l'analyse catégorielle a été retenue (Karsenti et Savoie-Zajc, 2011; Mucchielli, 1979). En l'absence de nouvelles catégories ayant émergé, la grille d'analyse se base essentiellement sur les catégories identifiées par l'étude de Karsenti et al. (2002) et celle de Mian Bi (2012). Elle a permis de traiter les informations récoltées et de catégoriser les différentes réponses dans des ensembles en fonction de leur signification et de leur interprétation selon le contexte.

\section{Résultats}

Lobjectif de la présente recherche est de mieux comprendre les usages que font des étudiants d'un groupe Facebook, comme instrument pour la médiation interpersonnelle (Rabardel, 1995), dans le cadre de leurs stages pratiques. Les deux premiers points de cette section abordent des éléments contextuels comme la présentation du groupe Facebook et les moyens utilisés par les étudiants pour y accéder. Les deux derniers nous permettent d'aborder la question des usages du groupe Facebook dans le cadre de leur stage et de son importance pour leur formation.

Pour les caractéristiques démographiques des répondants et la répartition géographique des lieux de stage, l'analyse des données du questionnaire adressé à tous les éducateurs fait ressortir que les répondants sont en majorité des hommes (71,9\%) dont l'âge varie de 28 à 38 ans. Pour leur stage, 
ces étudiants sont envoyés dans les établissements d'enseignement secondaire répartis sur toute l'étendue du territoire. En effet, les 32 stagiaires ont fait leur stage dans des établissements situés dans 20 départements (figure 1).

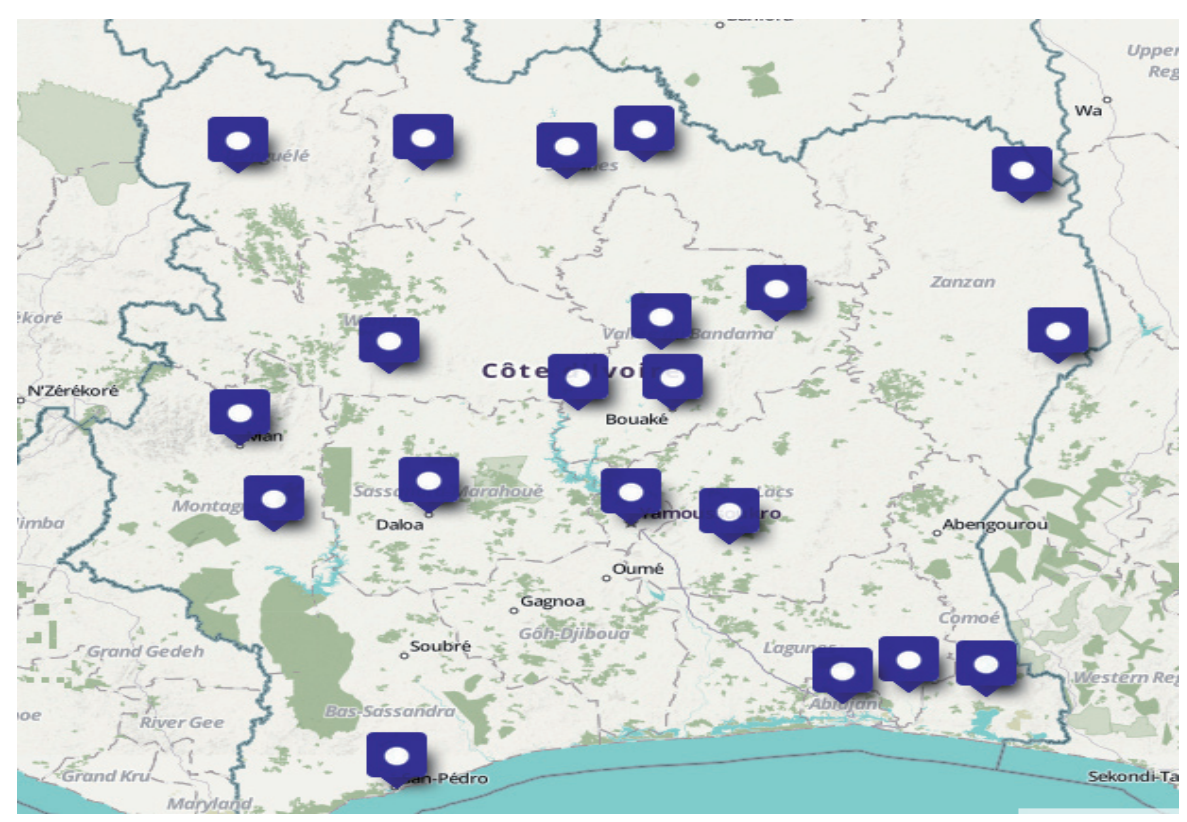

Figure 1

Répartition géographique des lieux de stage dans les départements de Côte d'Ivoire.

Présentation du groupe Facebook «ÉDUCATEURS PROMOTION 2014-2016 »

L'analyse des données qualitatives du guide d'entretien semi-directif adressé aux initiateurs du groupe Facebook fait ressortir qu'il a été créé « dans le courant du mois d'octobre 2014, soit quelques semaines après la rentrée académique à l'ENS d'Abidjan" et répond à un besoin de rester "constamment en contact les uns avec les autres ». Pour encourager l'inscription des autres collègues dans le groupe, les initiateurs du groupe les ont sensibilisés en faisant passer des annonces : "lors d'un cours en Amphithéâtre VakabaTouré,j'a i profité de la pause pour informer mes condisciples des sciences de l'éducation de la création du groupe. Ensuite, ceux d'entre nous qui étaient déjà sur les réseaux sociaux ont été ajoutés au forum ». À la question de savoir si le groupe avait un code de conduite élaboré, les initiateurs répondent "nous n'avons pas de code formellement établi. Cependant, chacun des membres observe les règles de bienséance et connaît la ligne éditoriale du groupe ». Toutefois, ils reconnaissent qu'il arrive des fois où certains membres partagent des publications qui ne cadrent pas avec l'esprit du groupe. Pour veiller au bon fonctionnement du groupe, quatre modérateurs et trois administrateurs ont été cooptés. 
Ces résultats semblent confirmer ceux de Mian Bi (2012) qui montraient que c'est la volonté de rester en contact qui poussait des étudiants à initier des groupes de travail sur Facebook. Dans ces groupes, même si les règles de gestion ne sont pas formalisées, les initiateurs, avec l'aide des autres membres, mettaient en place des systèmes de modération.

\section{Accès au groupe Facebook}

L'analyse des données quantitatives du questionnaire fait ressortir que pour accéder à Facebook (figure 2), les stagiaires se servent d'un téléphone intelligent («smartphone », 90,6\%), d'un ordinateur $(46,9 \%)$ ou d'une tablette $(6,3 \%)$.

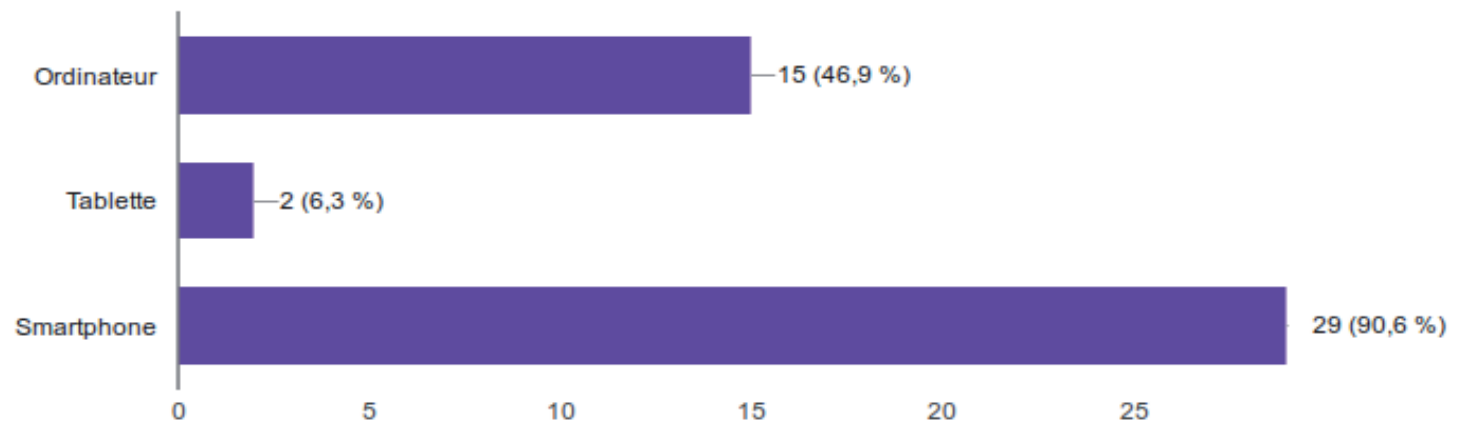

Figure 2

Équipements pour accéder à Facebook.

Les répondants accèdent à Facebook grâce à une connexion internet mobile (93,8 \%) ou une connexion internet ADSL (25 \%) (figure 3).

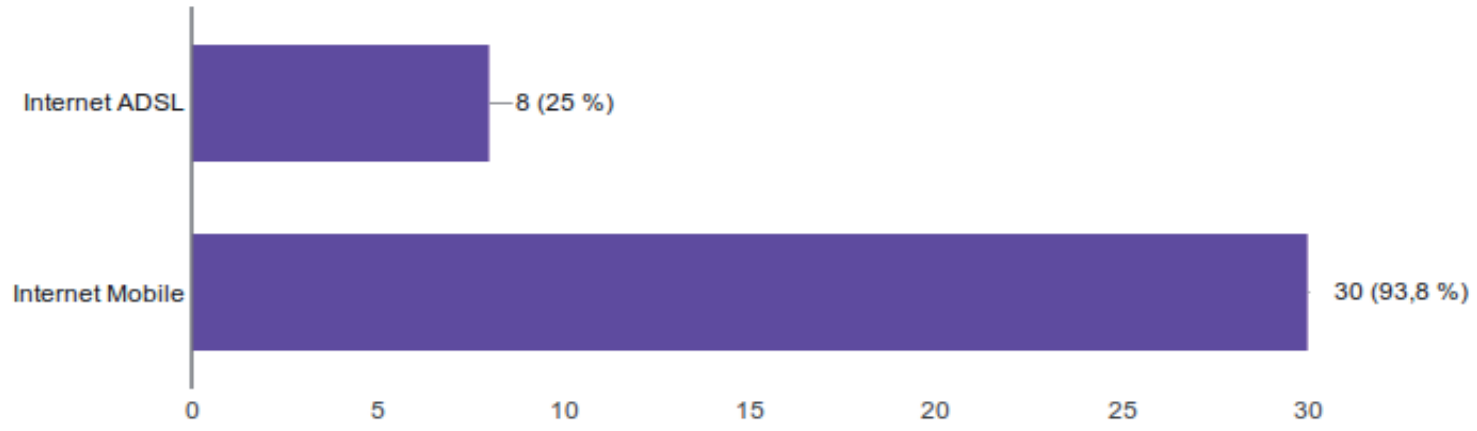

Figure 3

Type de connexion internet utilisé. 
À la question ouverte «comment ont-ils connu le groupe Facebook» issue du questionnaire, les répondants affirment que c'est par l'intermédiaire des initiateurs $(56 \%)$ ou des collègues $(46,9 \%)$ qui en parlaient. Ce chiffre est corroboré par les dires des initiateurs du groupe Facebook: "Lors d'un cours en Amphithéâtre Vakaba-Touré, j'ai profité de la pause pour informer mes condisciples des sciences de l'éducation de la création du groupe. Ensuite, ceux d'entre nous qui étaient déjà sur les réseaux sociaux ont été ajoutés au forum. J'ai également mis certains d'entre nous à contribution dans le cadre de la sensibilisation des réticents, notamment le délégué de flière, l'un des initiateurs du groupe. "

Pour accéder au groupe Facebook, les résultats montrent que les stagiaires utilisent majoritairement les terminaux mobiles et l'internet mobile. Pour faire connaître le groupe, initiative propre des étudiants, la sensibilisation semble le moyen privilégié comme l'a montré l'étude de Mian Bi (2012).

Les usages du groupe Facebook pendant le stage

La majorité des répondants (62,3 \%) accède au groupe Facebook plus d'une fois par jour et 37,5 \% affirment y accéder plusieurs fois par semaine. Ce qui les motive à fréquenter le groupe Facebook est la quête d'information sur leur formation. En effet, l'analyse des réponses de la question ouverte «Qu'estce qui vous motive à venir sur le groupe? " montre que 28 répondants sur les 32 affirment y venir pour accéder à des informations. Ceci est corroboré par les dires de ce répondant «Je viens sur le groupe pour avoir des informations sur la promotion et l'actualité de l'ENS d'Abidjan». Pour 15 répondants sur les 32, le groupe Facebook est un lieu de rencontre et d'échanges comme l'affirme celui-ci « Sur ce groupe, sans me déplacer, je rencontre des collègues avec qui nous échangeons des informations relativement à notre formation ». Sur les 32 répondants, 20 y viennent pour rester en contact avec des collègues. C'est cette idée de rester en contact qui a motivé la création du groupe comme l'affirment les initiateurs "nous avons été motivé par le besoin de rester constamment en contact les uns avec les autres ». Pour 13 répondants sur 32, le groupe Facebook est aussi un lieu de partage de connaissances et d'expériences comme l'indique ce répondant "j'y viens pour [...] le partage d'expériences». Ces résultats semblent mettre en exergue que Facebook devient un nouvel espace de socialisation (Loisier, 2014; Petiau, 2011) qui permet aux étudiants en stage de garder le contact avec le groupe (Asselin, 2002) et de s'informer dans le cadre de leur formation (Karsenti et al., 2002).

Si la majorité des répondants vient plus d'une fois par jour sur le groupe, ils ne sont que 37,5\% à partager souvent des publications (posts) dans le groupe Facebook. La majorité (62,5\%) partage moins souvent les posts dans le groupe. L'analyse des réponses à la question ouverte "Qu'est-ce qui vous motive à partager des posts dans le groupe? " montre qu'ils le font généralement pour donner des informations, partager des expériences et lancer des débats à travers des cas concrets rencontrés sur le terrain comme le soutient ce participant : "c'est toujours dans l'optique d'échanger nos expériences, et aussi permettre aux autres membres de donner leur avis". Il y a aussi le "besoin d'échanger sur les questions liées à la formation et à l'orientation", comme le soutient un autre répondant. Ainsi, les sujets généralement abordés par les répondants dans leurs posts (figure 4) portent sur le soutien et l'encouragement mutuel (75\%), l'échange d'expériences (71,9\%), les informations sur la formation (46,9\%) et le renforcement des acquis académiques (43,8 \%). 


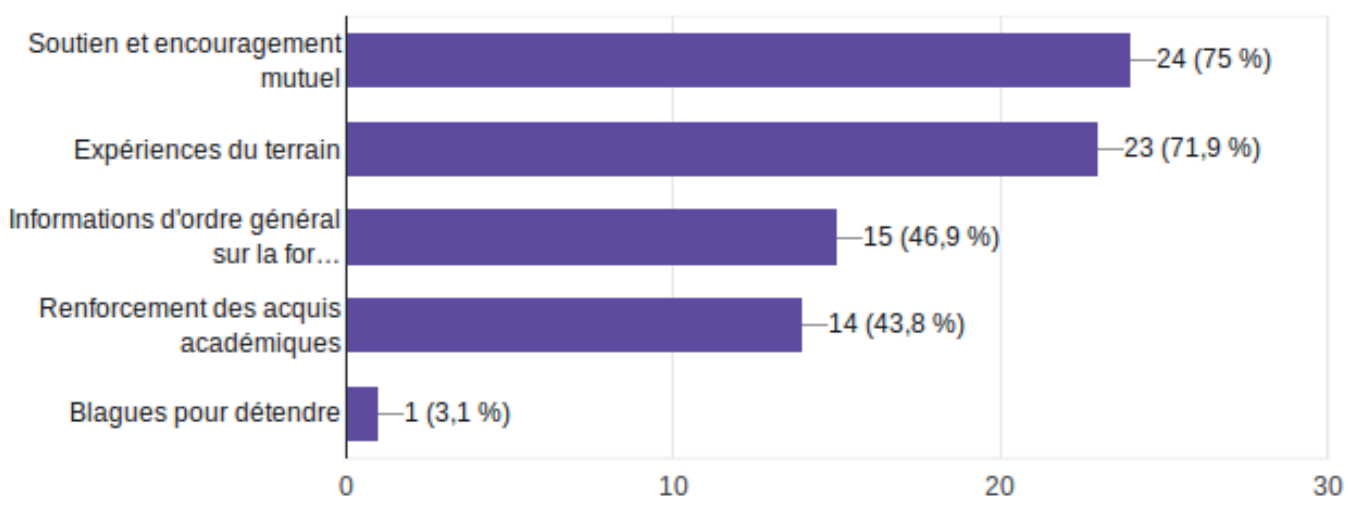

Figure 4

Sujets abordés par les étudiants dans le groupe Facebook.

En ce qui concerne les usages, 84,4 \% des répondants estiment que le groupe Facebook est un espace pour s'informer sur la formation et le déroulement du stage. Pour 78,1\% d'entre eux, il est un espace pour partager les expériences du terrain. Le groupe Facebook est aussi considéré par 71,9\% des répondants comme un espace de soutien et d'encouragement mutuel. Seulement 43,3\% des répondants estiment que le groupe Facebook est un espace de collaboration et de renforcement des acquis académiques. Les résultats semblent montrer que le groupe Facebook offre aux stagiaires un mode de soutien essentiel (Asselin, 2002; Karsenti et al., 2002). De plus, il permet le réseautage et un soutien personnel et professionnel (Mayer, 2002).

\section{Groupe Facebook dans la formation et la pratique professionnelle}

À partir de leurs expériences d'usage de Facebook dans le cadre de leur stage, les répondants estiment que Facebook peut jouer un rôle important au cours de la formation des éducateurs. L'analyse des réponses à la question ouverte « quel rôle pensez-vous que Facebook de façon générale et plus particulièrement le groupe Facebook peuvent jouer dans la formation? " montre que, pour 26 répondants sur 32, il peut être un outil de partage d'expériences et d'accès à l'information. Pour l'initiateur du groupe, "pendant le stage, le groupe a servi de véritable outil de diffusion d'informations et de partage d'expériences entre les éducateurs stagiaires. Nombreux sont ceux d'entre nous qui posaient des préoccupations liées aux activités pratiques dans le cadre du stage ». Cela est corroboré par l'affirmation de ce répondant : "De cette façon, Facebook est un moyen de partage d'expériences, de collaboration et de partage d'informations».

Conscients des potentialités du groupe Facebook, 29 répondants sur les 32 envisagent de l'utiliser dans l'exercice de leur profession. Ainsi, la question ouverte «Selon vous, comment Facebook peut-il aider l'éducateur dans sa fonction d'encadrement de la vie scolaire? », 20 répondants sur 32 répondent pour le partage d'expériences comme corroboré par cette réponse "partager les expériences réussies, mettre en valeur les expériences des élèves et critiquer souvent ». Sur les 32 répondants, 25 estiment qu'ils vont l'utiliser pour la communication et l'accès à l'information, car « les possibilités qu'offre Facebook en matière de communication sont énormes et également la majorité des apprenants s'intéresse à ces réseaux sociaux». Par ailleurs, ils pensent l'utiliser dans leur pratique professionnelle, parce que cet outil peut aider l'éducateur à accéder aux informations et à les partager, mais aussi à partager les expériences de terrain. 
Le groupe Facebook peut être un complément pour la formation des étudiants surtout en situation de stage. En effet, selon les résultats de l'analyse des données, il favorise un réseautage et un soutien personnel et professionnel (Asselin, 2002; Mayer, 2002) et le partage d'expériences (Karsenti et al., 2002). L'usage de cet outil peut être avantageux, car il permet de développer l'employabilité des étudiants en stage (He et al., 2017).

\section{Discussion}

Dans le contexte de la formation initiale à l'ENS d'Abidjan où des étudiants éprouvent souvent des difficultés lors des stages, l'objectif de la présente étude était de mieux comprendre les usages du groupe Facebook par des éducateurs, comme instruments de la médiation interpersonnelle en situation de stage. Alors que les réseaux sociaux sont très souvent présentés comme ludiques ou comme lieux d'échanges amicaux (Gokra, 2013) ou encore comme des moyens d'entretenir les relations amicales et faire passer le temps (Thivierge, 2011), la présente étude montre que Facebook peut être un outil au service de la formation en Afrique. En effet, les résultats font ressortir que le réseau social Facebook peut aussi être utilisé par des étudiants en stage pratique comme instrument de médiation interpersonnelle (Rabardel, 1995) pour favoriser leur intégration sociale (Tinto, 1975). Les résultats mettent ainsi en exergue que le détournement créatif et collectif de Facebook semble bénéfique pour des étudiants en situation de stage. En effet, en plus de leur permettre de garder le contact avec le groupe (Asselin, 2002), ce réseau social se révèle comme un outil permettant un réseautage et un soutien personnel et professionnel (Mayer, 2002). Mais contrairement aux travaux de Peraya et Bonfils (2014), de Lotfi et Hafedh (2016), et de Ben Rebah et Dabove (2017) où l'usage du groupe Facebook était une volonté de l'institution de formation, la présente étude met en exergue qu'en l'absence d'initiative institutionnelle, des étudiants en stage, de leur propre initiative, font usage des fonctions communicationnelles et informationnelles du groupe Facebook pour maintenir le contact et échanger des expériences. Toutefois, si cette prise d'initiative des étudiants est salutaire, elle n'est pas intégrée dans un véritable scénario pédagogique qui pourrait organiser le déroulement de l'activité dans le groupe Facebook (Ben Rebah et Dabove, 2017).

\section{Conclusion}

En matière de recherche, tout choix méthodologique présente toujours des limites. Le fait d'effectuer une étude par questionnaire sur une population de 32 étudiants dans un groupe Facebook qui en comptait plus de 200 peut être limitatif. Cette étude comporte aussi des limites, car l'analyse s'appuie en grande partie sur le discours des sujets. Conscient du fait que les analyses reposant sur le discours des sujets peuvent entraîner des oublis qui pourraient biaiser les résultats, nous avons, pendant plus d'une année, suivi les échanges entre les étudiants dans le groupe Facebook. Cette présence prolongée dans le groupe nous a permis de vérifier et de valider les discours des sujets. Par ailleurs, cette étude se fait en milieu naturel, car nous avons côtoyé les étudiants et vécu le quotidien des étudiants dans le groupe Facebook. 
Cette présence quotidienne peut, selon Laperrière (1997), crédibiliser la présente recherche dont les résultats montrent qu'au-delà d'être un simple outil de communication, Facebook peut être un vrai outil au service de la formation initiale. En effet, comme semblent le montrer les résultats de la présente recherche, dans le contexte actuel de la formation initiale à l'ENS d'Abidjan, le groupe Facebook permet à des étudiants en stage de rester en contact, de partager des expériences de terrain et de collaborer. Alors que la Côte d'Ivoire amorce un important virage dans l'intégration des TIC dans le système éducatif, les résultats de la présente étude semblent mettre en évidence le rôle important que pourrait jouer Facebook dans la formation initiale des personnels d'encadrement. Et pour un usage efficace de cet outil dans l'encadrement des stages de terrain, les résultats de l'étude suggèrent la mise en place de scénarios pédagogiques qui intègrent le groupe Facebook dans le cadre des stages pratiques. L'intégration de ce réseau social dans les scénarios pédagogiques en formation initiale à l'ENS d'Abidjan devra être faite en tirant des leçons des derniers déboires de Facebook sur la question de la protection des données personnelles et en tenant compte de l'entrée en vigueur de la réglementation européenne sur la protection des données.

À la suite de la présente étude qui semble mettre en exergue l'importance de Facebook comme outil en formation initiale à l'ENS d'Abidjan, une étude d'envergure portant sur l'amélioration de la qualité de la formation initiale par la mise en place des communautés de pratiques à travers les réseaux sociaux serait indiquée.

\section{Références}

Akoun, A. (2002). Nouvelles techniques de communication et nouveaux liens sociaux. Cabiers internationaux de sociologie, (112), 7-15. http://dx.doi.org/10.3917/cis.112.0007

Asselin, H. (2002). L'utilisation pédagogique d'outils de communication virtuelle durant le stage de fin d'études au collégial: un complément à la supervision directe. Repéré à https:/www.usherbrooke.ca/performa/fileadmin/sites/performa/ documents/Recherches_subventionnees/Asselin_2002_.pdf

Attenoukon, S. A., Coulibaly, M. et Karsenti, T. (2016). WhatsApp, un enjeu d'enseignement/apprentissage en Afrique? Enquête auprès des acteurs scolaires au Bénin. Transmettre, 1(3), 87-112. Repéré à http://www.karsenti.ca/Revue_Transmettre_3_Tire_a_part_Attenoukon_Coulibaly_Karsenti.pdf

Ben Rebah, H. et Dabove, G. M. (2017). Usage pédagogique de Facebook dans une activité d'apprentissage en groupe par des étudiants tunisiens : analyse de l'efficacité du travail collaboratif. Revue internationale des technologies en pédagogie universitaire, 14(1), 6-18. http://dx.doi.org/10.18162/ritpu-2017-v14n1-01

Berger, J. B., et Milem, J. F. (1999). The role of student involvement and perceptions of integration in a causal model of student persistence. Research in Higher Education, 40(6), 641-664. http://dx.doi.org/10.1023/A:1018708813711

Bogui, M. J.-J. (2007). Intégration et usages des technologies de l'information et de la communication (TIC) dans l'éducation en Afrique: Situation de l'enseignement supérieur en Côte d'Ivoire (2003-2005) (Thèse de doctorat, Université Michel de Montaigne - Bordeaux III, Pessac). Repéré à https://hal.archives-ouvertes.fr/tel-00265498

Chomienne, E. et Lehmans, A. (2012). Réseaux sociaux et apprentissages collaboratifs à l'université : pratiques innovantes dans une communauté connectée. Repéré à https://hal.archives-ouvertes.fr/hal-00688562

Diakhaté, D. et Akam, N. (2015, novembre). L’usage du réseau social Facebook dans la coconstruction des connaissances chez les étudiants. Communication présentée au congrès international «Les écosystèmes numériques et la démocratisation informationnelle : intelligence collective, développement durable, interculturalité, transfert de connaissances », Schœlcher. Repéré à https://hal.archives-ouvertes.fr/hal-01258319v1 
Gérard, J.-P. (2007). Formation des enseignants : réseau social en présentiel vs réseau social virtuel. Une plateforme de travail collaboratif, outil de construction de relations sociales différentes. Revue Expressions, (32), 179-212. Repéré à https://espe.univ-reunion.fr/fileadmin/Fichiers/ESPE/bibliotheque/expression/32/Gerard.pdf

Gokra, D. A. O. J. (2013). Médias ou réseaux sociaux : un point de vue africain. Repéré à http://www.academia. edu/4197146/Médias_ou_réseaux_sociaux_un_point_de_vue_africain

He, C., Gu, J., Wu, W., Zhai, X. et Song, J. (2017). Social media use in the career development of graduate students: the mediating role of internship effectiveness and the moderating role of Zhongyong. Higher Education, 74(6), 10331051. http://dx.doi.org/10.1007/s10734-016-0107-8

Karsenti, T., Lepage, M. et Gervais, C. (2002). Accompagnement des stagiaires à l'ère des TIC : forum électronique ou groupe de discussion? Formation et profession, 8(2), 7-12. Repéré à https://www.researchgate.net/ publication/267035243 ccompagnement des stagiaires a $1 \% 27 \mathrm{ere}$ des TIC forum electronique ou groupe de discussion

Karsenti, T. et Savoie-Zajc, L. (2011). La recherche en éducation :étapes et approches (3éd.). Montréal, QC : ERPI.

Koffi, G. E. E. (2007). Formation initiale des enseignants à l'ENS d'Abidjan : un exemple d'appropriation culturelle et cognitive des TICE. Agence universitaire de la Francophonie.

Koffi, K. A. (2012). Motivations et difficultés de stagiaires en formation d'éducateurs à l'ENS. African Education Development Issues, (4), 61-85. Repéré à http://www.rocare.org/aedi4/ch3-AEDI4.pdf

Krou Adohi, V. (2012). La réforme LMD en Côte d'Ivoire : Mise en cuvre et enjeux. Repéré à http://enseignement.gouv.ci/ fichiers/mise_en_oeuvre_enjeux.pdf

Laferrière, T., Bracewell, R., Breuleux, A., Erickson, G., Lamon, M. et Owston, R. (2001). La formation du personnel enseignant ceuvrant dans la classe en réseau. Communication présentée au Colloque du Programme pancanadien de recherche en éducation, Québec, QC. Repéré à https://web.archive.org/web/20160805234617/http://www. cesc.ca/pceradocs/2001/papers/01Laferriere_etal_f.pdf

Laperrière, A. (1997). Les critères de scientificité des méthodes qualitatives. Dans J. Poupart, J.-P. Deslauriers, L.H. Groulx, A. Laperrière, R. Mayer et A. P. Pires (dir.), La recherche qualitative : enjeux épistémologiques et méthodologiques (p. 365-388). Montréal, QC : Gaëtan Morin.

Loisier, J. (2014). La socialisation des étudiants en FAD au Canada francophone. Repéré à http://www.refad.ca/wp-content/ uploads/2014/04/Guide_sur_la_socialisation_en_FAD.pdf

Loiseau, M., Potolia, A. et Zourou, K. (2011). Communautés Web 2.0 d'apprenants de langue avec parcours d'apprentissage : rôles, pédagogie et rapports au contenu. Dans M. Bétrancourt, C. Depover, V. Luengo, B. De Lièvre et G. Temperman (dir.), Actes du colloque Environnements informatiques pour l'apprentissage humain (EIAH 2011) (p. 111-123). Repéré à https://hal.archives-ouvertes.fr/hal-00598762v3

Lotfi, B. A. et Hafedh, B. (2016). Les réseaux sociaux dans l'enseignement supérieur : un outil de partage et de coordination. Cas des groupes fermés de la formation à distance. Repéré à http://www.academia.edu/25480756/Les r\%C3\%A9seaux sociaux dans lenseignement sup\%C3\%A9rieur un outil de partage et de coordination. Cas des groupes ferm\%C3\%A9s de la formation \%C3\%A0 distance

Loukou, A. F. (2015). Niveau de diffusion des TIC dans les établissements d'enseignement de la ville de Bouaké et application du concept «TIC en éducation ». Germivoire, (2), 210-225. Repéré à https://web.archive.org/ web/20170329063937/http://www.germivoire.net/download/160104031931.pdf

Mayer, D. (2002). An electronic lifeline: information and communication technologies in a teacher education internship. Asia-Pacific Journal of Teacher Education, 30(2), 181-195. http://dx.doi.org/10.1080/13598660220135685

Mélot, L., Strebelle, A., Mahauden, J. et Depover, C. (2016). Le réseau social Facebook comme support d'apprentissage pour les étudiants universitaires. Dans S. George, G. Molinari, C. Cherkaoui, D. Mammas et L.Oubahssi (dir.), Actes de la $7^{e}$ Conférence sur les Environnements informatiques pour l'apprentissage humain (ELAH 2015) (p. 102-113). Repéré à https://hal.archives-ouvertes.fr/hal-01405938 
Mian Bi, S. A. (2012). Usages de Facebook pour l'apprentissage par des étudiants de l'Institut Universitaire d'Abidjan (IUA). Repéré à http://www.adjectif.net/spip/spip.php?article142]

Mian Bi, S. A. (2016). Le profil TIC des personnels d'appui technique à l'éducation en formation initiale à l'ENS d'Abidjan. Dans T. Karsenti (dir.), Mieux former les enseignants dans la Francophonie. Principaux enjeux actuels et futurs (p. 6-17). Montréal, QC : AUF.

Mucchielli, R. (1979). L'analyse de contenu des documents et des communications. Paris : ESF.

Nault, T. et Nault, G. (2001). Quand les stages attrapent les TIC. Dans T. Karsenti et F. Larose (dir.), Les TIC... au cœur des pédagogies universitaires (p. 145-164). Sainte-Foy, QC : Presses de l'Université du Québec.

Nogry, S., Decortis, F., Sort, C. et Heurtier, S. (2013). Apports de la théorie instrumentale à l'étude des usages et de l'appropriation des artefacts mobiles tactiles à l'école. Sciences et technologies de l'information et de la communication pour l'éducation et la formation (STICEF), (20), 413-443. http://dx.doi.org/10.3406/stice.2013.1077

Peraya, D. et Bonfils, P. (2014). Détournements d'usages et nouvelles pratiques numériques : l'expérience des étudiants d'Ingémédia à l'Université de Toulon. Sciences et technologies de l'information et de la communication pour l'éducation et la formation (STICEF), (21), 239-268. http://dx.doi.org/10.3406/stice.2014.1098

Petiau, A. (2011). Internet et les nouvelles formes de socialité. Vie sociale, (2), 117-127. http://dx.doi.org/10.3917/vsoc.112.0117

Plantard, P. (2016). Anthropologie des usages des technologies numériques. Champs culturels, (28).

Rabardel, P. (1995). Les hommes et les technologies, approche cognitive des instruments contemporains. Paris : Armand Colin.

Tchameni Ngamo, S. (2007). Stratégies organisationnelles d'intégration des TIC dans l'enseignement secondaire au Cameroun : étude d'écoles pionnières (Thèse de doctorat, Université de Montréal). Repéré à https://papyrus.bib.umontreal.ca/xmlui/handle/1866/17687

Thivierge, J. (2011). Jeunes, TIC et nouveaux médias : une étude exploratoire au Cégep de Jonquière. Repéré à https://ecobes. cegepjonquiere.ca/media/tinymce/Publication-Education/RappNvMedias_ELECTRONIQUE_ 11Nov11.pdf

Tinto, V. (1975). Dropout from higher education: A theorical synthesis of recent research. Review of Educational Research, 45(1), 89-125. http://dx.doi.org/10.2307/1170024

Vergnaud, G. (1991). La théorie des champs conceptuels. Recherches en didactique des mathématiques, 10(2-3), 133-170.

\section{Pour citer cet article}

Mian Bi, S. A. (2019). Usages du groupe Facebook en situation de stage : le cas des éducateurs de l'ENS d'Abidjan. Formation et profession, 27(1), 70-83. http://dx.doi.org/10.18162/fp.2018.488 